\title{
Emprendimiento en la Tercera Edad: Una Revisión de la Situación Actual
}

\author{
Felipe Oelckers ${ }^{1 *}$
}

\begin{abstract}
A medida que la población envejece, los países han empezado a comprender la importancia del emprendimiento de la tercera edad como un mecanismo para extender la vida laboral, aumentar los ingresos y generar nuevas oportunidades, a fin de mantener a los adultos mayores activos, situación que significa, a su vez, un menor peso para la carga fiscal del país.

Este trabajo tiene como objetivo hacer una revisión de la literatura sobre el emprendimiento en la tercera edad, analizar las oportunidades y los obstáculos que enfrenta este creciente sector de la población. Asimismo, se exploran las razones y condiciones que determinan las causas por las que este fenómeno llegará, con el paso del tiempo, a tener una mayor importancia en la sociedad chilena.
\end{abstract}

Keywords: tercera edad, oportunidades, emprendimiento, barreras, envejecimiento, población.

Abstract: As the population ages and the retirement age increases, countries have begun to realize the importance of senior entrepreneurship, as a means to extend the working age, increase income for the elder, especially those with low pensions and to generate opportunities to keep this people economically active.

This paper aims to review the literature of elderly entrepreneurship, analyzing the opportunities and obstacles being faced by this sector of the population. The reasons and conditions that have made this phenomenon increasingly important to our society in Chile are also explored.

Keywords: Third age, opportunities, entrepreneurship, barriers, aging, population.

Submitted April 23rd 2015/ Approve October 7th 2015

\section{Introducción}

Existen un sinnúmero de antecedentes que indican que la población mundial está envejeciendo (OECD, 2012). Entre los años 2000 y 2050 la proporción de la población mundial con más de 60 años de edad se duplicará, pasará de aproximadamente el 11\% al 22\% (OMS, 2012). Sin perjuicio de lo anterior, se espera que el número de personas de 60 años o más, aumente de 605 millones a 2000 millones en ese mismo periodo (OMS, 2012). Por otro lado, tanto en países desarrollados, como algunos en desarrollo, las tasas de natalidad han empezado a reducirse en forma muy rápida y las expectativas de vida han aumentado (Weber y Schaper 2004). En diversos países del mundo, tales como Italia y Japón, entre otros, existe una amplia población de adultos mayores dependientes de personas que pagan impuestos para sus pensiones (OECD, 2012).

De este modo, el aumento de la expectativa de vida en las economías desarrolladas, ha tensionado los recursos fiscales de las naciones (OECD 2001; OECD 2006). De hecho, los estados han tenido que realizar una serie de reformas para extender la edad de retiro y traspasar la responsabilidad y financiamiento de la jubilación a las propias personas (Wainright y Kibler, 2013). Actualmente en el Reino Unido, se ha hecho el ejercicio de calcular el ingreso a percibir bajo la hipótesis que cada adulto mayor retrasara su jubilación y trabajara un año más. El resultado obtenido fue una especial contribución a la economía británica del orden de 13 billones de libras esterlinas (BIS, 2011). Ante este escenario, las personas de la tercera edad y adultos mayores emprendedores, tendrán un rol muy significativo en las actividades económicas del futuro (OECD, 2012). Los estudios realizados por Zhang (2008), concluyeron que los emprendedores de la tercera edad se jubilan más tarde que los empleados dependientes del mismo grupo etario. De hecho, el emprendimiento en adultos mayores ha estado desde hace muy poco tiempo concebido como políticas sociales y públicas destinadas a potenciar el desarrollo de nuevos ingresos económicos, cuando las pensiones son limitadas y existen escasas oportunidades para participar en el mercado laboral. (Webster y Walker 2005; Kautonen, 2012). Estas políticas pueden constituir un potencial remedio para enfrentar el proceso de envejecimiento acelerado de la población. (Maestas y Zissimopoulos, 2010).

Cabe destacar, asimismo, que los adultos mayores deben enfrentar una serie de barreras para lograr emprender (Loretto, 2010, Semana 2013). Actualmente, la edad y el estado de salud, constituyen elementos que están afectado negativamente las actividades empresariales de los adultos mayores (Kibler et al., 2011). Además, estas personas tienen numerosas probabilidades de experimentar discriminación por su edad en el momento de generar actividades empresariales (Dibden

1 Escuela de Negocios, Universidad Adolfo Ibáñez, Viña del Mar, Chile,

*Email: felipe.oelckers@uai.cl 
y Hibbett, 1993). Cabe agregar que la proporción de adultos mayores que están comenzando un emprendimiento, constituye la mitad de los jóvenes en la misma situación (Hart et al., 2004; Kautonen 2008) y el panorama empeora en comparación con el adulto mayor femenino (Mckay, 2001; Kautonen 2012; OECD 2012).

Por otro lado, en Australia, alrededor de un tercio de los dueños de empresas tienen 50 años o más y su número aumenta cada año, situación que contrasta con personas de menores edades. Este fenómeno ocurre también en forma evidente en muchos otros países del mundo (Weber y Schaper, 2004). De hecho, estudios desarrollados por Kautonen et al. (2009) concluyeron que los adultos mayores poseen más habilidades técnicas y administrativas que los emprendedores jóvenes. Además, las personas mayores, tienen los beneficios de la experiencia laboral (Kibler et al., 2011), situación que les permite enfrentar de mejor manera los problemas propios de un nuevo negocio. En efecto, es posible encontrar en ellos un profundo conocimiento de una industria, situación que normalmente no poseen los jóvenes. Lo anterior puede ayudar a la persona a identificar las brechas para crear nuevos negocios y aprovechar una oportunidad. (Kautonen, 2012). Sin embargo, numerosos adultos mayores en países desarrollados deciden no emprender, en consideración a que los beneficios estatales vinculados a ingresos por jubilación son suficientemente buenos. Dicha situación provoca desincentivos a desarrollar un negocio, perdiendo el conocimiento, la experiencia y el talento desarrollados en años anteriores (Halabisky 2012).

Por esta razón, entender y analizar el fenómeno expuesto y diseñar políticas públicas que apoyen el emprendimiento para este grupo etario, constituye un enorme reto que podrán enfrentar los países en los próximos años (Weber y Schaper, 2004). De hecho, la falta de datos empíricos (Kautonen, 2008) en esta área hace que las investigaciones del emprendimiento de personas de la tercera edad se manifieste como una motivación y un desafío para la investigación.

\section{Envejecimiento de la población en Chile y empeoramiento de las condiciones económicas}

Chile es el segundo país más envejecido de Latinoamérica, después de Uruguay (Canals, 2012). Actualmente el 15,6\% de la población son considerados adultos mayores y para el año 2025 se espera que sean más del 20\% (Senama, 2013). Hoy en día, el 57\% de los adultos mayores se encuentran en calidad de jubilados y muchos de ellos aún están en condiciones de autovalencia física y cognitiva para seguir económicamente activos (Senama, 2013). La gran mayoría de los adultos mayores jubilados en Chile obtienen sólo un tercio de los ingresos que percibían en su vida laboral activa (Senama, 2011). Muchos de ellos se encuentran en desventaja económica, puesto que antes de cumplir la edad de jubilar los deponen de sus trabajos, entrando en situación de pobreza (Loretto, 2010). De hecho, en Chile la discriminación de edad, impide a estas personas las posibilidades de acceder a créditos o desarrollar un trabajo dependiente (Kornfeld, 2013).

El desarrollo del emprendimiento, podría constituir en Chile una buena alternativa para contrarrestar la falta de oportunidades laborales en los adultos mayores. Sin embargo, el emprendimiento del adulto mayor no ha sido considerado en este país como una política pública clave a pesar de que el Estado promueve la actividad emprendedora.

La solución expuesta, además de potenciar el emprendimiento en el adulto mayor, permitiría contener por sobre la línea de pobreza a aquellas personas que tienen bajas pensiones o bien que no tienen una jubilación.

Vista la preponderancia que tendrá en el futuro este grupo etario en la sociedad chilena, considerar el emprendimiento del adulto mayor y fortalecer su desarrollo adquiere vital importancia como alternativa viable, no solo para el mejorar los ingresos y calidad de vida de las personas mayores, sino también, con el objeto de apoyarlos en el cambio psicológico que experimentan al sentirse valiosos y útiles a la sociedad, aportando sus conocimientos y experiencia al encontrar nuevamente un sentido para sus vidas.

\section{Revisión de Literatura}

\section{Definición de emprendedor de la tercera edad}

Arkebauer (1995) establece que los emprendedores de la tercera edad son aquellos que tienen sobre 50 años y más, y que poseen algún negocio sin importar su tamaño. Por otro lado, Baucus y Human (1994) se refieren a emprendedores de la tercera edad como aquellos que han emprendido negocios con más de 50 años, excluyendo los negocios generados en el pasado. A su vez, Blackburn et al. (2000) han definido a los emprendedores de la tercera edad, como individuos que operan un negocio con edad entre 50 y 75 años y que además hayan fundado el negocio con 50 años o más. En general, en investigaciones más recientes, este término se refiere a individuos con 50 años o más, que están en el proceso de comenzar un negocio o ya han comenzado uno (Kautonen, 2012). Sin embargo, no existe una definición absoluta y para efectos de este trabajo se tomará como emprendedores de la tercera edad en Chile, a aquellas personas que están activamente tratando de comenzar un negocio, han comenzado uno o bien son auto empleados (Levie, 2014) con 60 años o más.

En la literatura actual, se han identificado los siguientes temas para explicar las principales líneas de investigación que se han desarrollado para estudiar el fenómeno del emprendimiento del adulto mayor: Ambiente cultural y contexto emprendedor (Weber y Schaper, 2004; Kautonen,et al.,2009; Pilkova, el al.,2014); Acceso financiero y otros recursos (Hart et al., 2004; Kibler et al., 2011), Barreras para emprender (Curran y Blackburn, 2001; Singh y DeNoble, 2003, Levesque y Minniti, 2006; Kautonen et al., 2011; Kibler, et al., 2011, Kibler et al., 2012, Kautonen, et al., 2013), Capital humano (Singh y DeNoble, 2003; Weber y Schaper, 2004), Capital social (Baucus y Human, 1994; Singh y DeNoble, 2003; Kautonen, 2012), Historia de carrera (Baucus y Human, 1994; Kautonen et al., 2010), Emprendimiento femenino (McKay, 2001; Kautonen, 2008; Kautonen, 2012), Innovación (Botham y Graves, 2009; Colovic y Lamotte, 2012) Jubilaciones y emprendimiento (Wainright y Kibler, 
2014), Motivaciones para emprender (Weber y Schaper, 2004; Kautonen et al., 2008; Kibler et. al., 2012, Kautonen et al., 2013) y finalmente Políticas Públicas (Kautonen, 2012; Halabisky 2012)

A su vez, dado que cada una de las diferentes líneas de investigación descritas toman distintas perspectivas, se ha decido hacer una clasificación que aborda temas más amplios, con el fin de estudiarlos en mayor profundidad y claridad. A continuación se procederá a explicar cada una de estas materias.

\section{Factores que están potenciando el emprendimiento de la tercera edad.}

Motivación, predisposición y desarrollo innovador.

Debido a los cambios socio-demográficos que se están generando, los adultos mayores están presionados "push" o bien atraídos "pull" a emprender. En relación al factor "push" los adultos mayores se ven presionados a emprender por varias razones: se encuentran desempleados, no pueden obtener beneficios sociales, tienen bajas pensiones, existe disconformidad laboral o bien tienen bajos salarios (Webster y Walker, 2005). De acuerdo a estudios desarrollados por McClelland et al. (2005) las personas a medida que envejecen van cambiando sus valores y visión de la vida, sumado a la insatisfacción con su actual situación laboral y la falta de oportunidades en el mercado laboral, genera la decisión de embarcarse en un emprendimiento por necesidad.

Por otro lado, existen factores "pull" que atraen a los adultos mayores a desarrollar un negocio. Estas causas son múltiples y de variado contenido, tales como: encontrar una oportunidad, buscar independencia, dejarle una herencia a sus hijos, flexibilidad laboral y ser su propio jefe (Karoly y Zissimopoulos, 2004; Webster y Walker, 2005). Estudios empíricos sobre la predisposición a emprender por parte de adultos mayores concluyen, que esta depende de la experiencia y carrera laboral desarrollada (Weber y Schaper, 2004). Haber tenido experiencia emprendedora o en actividades de gestión empresarial ocasiona que la predisposición a comenzar un negocio sea mayor (Kautonen et al., (2010). Lo dicho se confirma con estudios realizados también por Kautonen et al. (2013) al concluir que la relación entre la edad y el compromiso de los adultos mayores para emprender depende en gran medida del historial de la carrera desarrollada en el pasado.

Si consideramos estudios empíricos, podemos afirmar que estos han concluido que el emprendimiento del adulto mayor no necesariamente está relacionado con emprendimientos innovadores (Pilkova et al., 2014), pero efectivamente constituye una opción válida para aumentar las pensiones, extender la vida laboral y contrarrestar la falta de oportunidades laborales (Kautonen, et al., 2008). Aunque muy poco extendida e investigada, la capacidad de innovación de la tercera edad aún no es una fuente importante de creación de nuevos negocios, pero en el futuro se espera que sea un significativo factor para potenciar el emprendimiento en este grupo etario (Botham y Graves, 2009).

\section{El emprendimiento como una alternativa a una pensión insuficiente.}

De acuerdo con Wainwright y Kibler (2013), el emprendimiento de la tercera edad tiene un particular interés de complementar las bajas pensiones, o bien, proveer de alguna renta adicional cuando no se cuenta con una jubilación adecuada. Este nuevo modelo se explica a partir de un retiro híbrido, donde las personas además de recibir una jubilación, en el caso de que la tengan, obtienen un ingreso a partir de un emprendimiento desde sus propios hogares (Clark, Munnell y Orszag, 2006; Wainwright y Kibler, 2013).

Es así como el emprendimiento del adulto mayor pasa a ser un modelo de retiro, principalmente a personas de la tercera edad que tienen bajas pensiones, debido a la dificultad de ahorro en el pasado por sus bajos salarios y también al escaso conocimiento financiero. (Munnel, Sunden y Taylor, 2002). Cabe agregar, que en el caso de aquellas personas que poseen mejores salarios y lograron ahorrar más durante su vida laboral activa, la volatilidad de los mercados financieros no aseguran tampoco un ingreso adecuado en el largo plazo (Froud et al., 2002). Por tanto, este modelo logra tener aún más sentido (Wainwright y Kibler, 2013).

\section{Capital humano, social y acceso a recursos financieros.}

La personas de la tercera edad, sin duda, poseen los beneficios de contar con experiencia laboral, buenas habilidades administrativas y técnicas para poder emprender (Singh y DeNoble, 2003; Weber y Schaper, 2004; Kautonen et al., 2008). Los adultos mayores y con experiencia emprendedora desarrollada en el pasado tienen más posibilidades de comprometerse con nuevos emprendimientos en comparación a adultos mayores que trabajaron en actividades con bajos sueldos y que requerían poca preparación y habilidades (Fuchs, 1982; Kautonen et al., 2010).

Por otro lado, investigaciones confirman que el capital social está fomentando el emprendimiento de la tercera edad (Pilkova et al., 2014). Los adultos mayores que poseen en general amplias redes de contacto pueden facilitar y ayudar para el desarrollo y operación de un nuevo negocio (Bacus y Human, 1994; (Bruin y Firkin, 2001). Sin embargo, las redes para los adultos mayores empiezan a declinar con el tiempo en la medida que las personas se van retirando (Botham y Graves, 2009).

Singh y DeNoble (2003) en sus investigaciones concluyeron que los recursos financieros acumulados durante la vida laboral pueden materializarse en una fuente importante de ingresos destinados al apoyo de emprendimientos en etapas tardías. En la medida que las personas envejecen, aumenta la disposición a tomar más riesgos principalmente financieros puesto que ya no tienen hijos dependientes en materia económica (Kiber et al., 2011). Además hay estudios concluyentes respecto a que los adultos mayores de países desarrollados con recursos financieros logran mayor compromiso para desarrollar emprendimientos (Webster y Walker, 2005; Singh y DeNoble, 2003; Kibler et al., 2011). 
El contexto del emprendedor y las instituciones relacionadas.

El contexto emprendedor y las instituciones cumplen un rol muy importante en el desarrollo del emprendimiento de la tercera edad (Mrva y Stachova, 2014, Pilkova et al., 2014). La teoría de instituciones, de acuerdo con North (1990), representa las reglas de juego en una sociedad y determinan las acciones que desarrollan los agentes en una economía. De acuerdo con North (1990), existen dos tipos de instituciones: las formales e informales. El primer concepto tiene relación con las leyes, el marco legal y jurídico que regula la conducta social. El segundo concepto se refiere al ambiente social que tiene relación con las limitaciones originadas en la sociedad que son parte de la cultura.

En relación a las instituciones formales, estudios de Pilkova et al. (2014) concluyeron que existen diferencias significativas entre los países del este de Europa que poseen altos niveles de emprendimiento de la tercera edad y aquellos países que no lo poseen, fundamentalmente por las instituciones que apoyan este fenómeno. Las políticas concretas de soporte vienen dadas por apoyar en áreas relacionada con la burocracia e impuestos para la puesta en marcha de un negocio (Pilkova et al., 2014).

Investigaciones realizadas por Kautonen et al. (2009) sobre el ambiente social, concluyeron que este factor juega un rol muy importante para potenciar el emprendimiento en la tercera edad. Es así como el entorno familiar y de los amigos más cercanos pueden facilitar o bien obstaculizar el proceso de puesta en marcha de un negocio. Los ambientes de familiares y amigos que han tenido experiencia emprendedora pueden apoyar y fomentar el desarrollo emprendedor en la tercera edad (Kautonen et al., 2009). Varios estudios sobre la cultura y el emprendimiento del grupo etario que es materia de este trabajo, confirman que la apertura cultural de una sociedad hacia los adultos mayores tiene efectos positivos para que estas personas logren involucrarse en actividades emprendedoras (Weber y Schaper, 2004; Kautonen et al., 2011).

\section{Envejecimiento activo y educación emprendedora}

Este tema posee gran importancia, al punto que la Comisión Europea declaró el 2012 como el año del envejecimiento activo. De acuerdo con investigaciones de Kibler et al. (2012), las personas de la tercera edad buscan un envejecimiento dinámico, siendo el emprendimiento una herramienta que les permite tener un balance de trabajo, ingresos y tiempo libre. De acuerdo con Kibler et al. (2012), la búsqueda de este equilibrio se manifiesta fundamentalmente en dos aspectos: individual y social. El primero tiene relación con la identidad personal de mantenerse con actividades que los mantengan ocupados, generar ingresos para complementar la pensión y desarrollar sus propios intereses para promover sus valores personales en los productos o servicios que venden o que podrían vender. El segundo, se refiere a compatibilizar las responsabilidades empresariales con custodia de nietos, cuidados a familiares enfermos y voluntariado.

A su vez, este concepto se vincula con la educación emprendedora que ha empezado a tener cada vez más importancia. De acuerdo con Fernández y Rey (2010), se está asentando una estrategia política y legislativa que determina con claridad el valor del fomento del espíritu emprendedor. Se refiere a que dicha política sería apta para incorporar la cultura emprendedora desde el principio de la educación hasta los últimos eslabones del sistema educativo, sin ignorar tampoco a los adultos mayores (Fernández y Rey, 2010).

\section{Barreras que afectan al emprendimiento del adulto mayor}

\section{Envejecimiento y discriminación por edad: un problema en aumento}

Según Kibler et al. (2011), la edad es una de las barreras más importantes que afectan el desarrollo empresarial en la tercera edad. Según estudios desarrollados por Weber y Schaper (2004); Kautonen et al. (2009); McKay (2011) para muchos emprendedores adultos mayores, la edad no es una barrera para emprender, ya que muchos se sienten más jóvenes de lo que realmente son, sin embargo, perciben una discriminación de edad, como una barrera externa a ellos, que los dificulta para tener un emprendimiento. Esta discriminación de edad no ocurre en forma abstracta, ya que acontece en forma muy directa. (Kibler et al., 2011). Estudios desarrollados por Kautonen (2012) concluyen que la sociedad aún mantiene la percepción que los adultos mayores no deben involucrarse en actividades económicas y laborales. Además, son vistos como menos flexibles, menos comprometidos y menos capaces de hacer frente a cambios tecnológicos. Estudios realizados por Kibler et al. (2012) establecieron que algunos clientes cuestionan las habilidades de los adultos mayores para proveer de bienes y servicios debido a la edad. Este hecho es percibido por ellos como impedimento para aprovechar oportunidades y desarrollar un negocio (Kibler et al., 2012). La referida situación empeora en el colectivo de adultos mayores femenino. Actualmente, existen muy pocos emprendimientos de la tercera edad desarrollado por mujeres (Halabisky, 2012). Los estudios realizados por McKay (2001) concluyen que la sociedad aún mantiene la idea de que las mujeres de la tercera edad deben estar involucradas en el cuidado de los miembros de la familia, más que en la terea de emprender. De hecho, McKay (2001) concluye que las responsabilidades de cuidados familiares tienen un efecto importante en los emprendimientos de las adultas mayores, tanto en la fase de fundación de la empresa como en etapas posteriores. Según estudios de Hart et al. (2004) las mujeres de la tercera edad tienen menos posibilidades de emprender en comparación a los adultos mayores hombres.

\section{Capital humano, social y acceso a recursos financieros.}

Generalmente, aquellos adultos mayores que comienzan con actividades emprendedoras lo hacen en negocios relacionados con los trabajos remunerados hechos en el pasado (Halabisky, 2012). Sin embargo, enfrentan problemas de escasez de habilidades emprendedoras para superar etapas preliminares de un negocio (Kautonen, 2012). Además, en algunos casos, sus habilidades están obsoletas y tienen dificultades para desarrollarse profesionalmente, especialmente al no poder utilizar las nuevas tecnologías. (Kadefors, 2011). Estudios han demostrado que el tipo de trabajo que haya desarrollado una persona, durante su vida activa, determinará si puede llegar a ser un emprendedor de la tercera edad y adquirir nuevas habilidades y conocimientos (Kibler et al., 2012). 
De acuerdo a investigaciones de Kautonen (2012), el legado de una cultura de trabajo tradicional parece formar una barrera para un comportamiento más emprendedor. Los hombres que tuvieron puestos de trabajo de industria tradicional y que se sentían orgullosos de tenerlos, son muy reacios a aceptar nuevos tipos de trabajo o emprender una nueva actividad. Este hallazgo coincide con investigaciones previas, en que el historial de carrera de un individuo tiene un impacto significativo en la probabilidad de reinventarse para ser empresario en una edad mayor. (Fuchs, 1982; Kautonen et al, 2010)

Cabe mencionar que estudios empíricos desarrollados por Kibler et al. (2011) han concluido que el capital social es una barrera para poder emprender. En efecto, los adultos mayores que se encuentran jubilados manifiestan dificultades para el desarrollo de nuevas redes de contactos, principalmente cuando proyectan un pequeño negocio desde sus casas. Sin perjuicio de lo anterior, se presenta el interesante desafío para desarrollar nuevas habilidades sociales, diferentes a las conocidas en el pasado (Halabisky, 2012), principalmente cuando el emprendimiento tiene un giro diferente a la actividad laboral desarrollada en el último trabajo. Esto se complica aún más, cuando las redes de contactos quedan obsoletas porque los individuos han pasado mucho tiempo desempleados o jubilados (Kibler et al., 2012). De acuerdo con Kibler et al. (2011), las barreras que enfrentan los adultos mayores para conseguir recursos financieros para comenzar un negocio no son sustancialmente diferentes en comparación con otros emprendedores. Según Kautonen (2008), el acceso a recursos financieros se hace mucho más complejo para aquellos adultos mayores que están desempleados o que reciben bajas pensiones en comparación a individuos de la tercera edad que están trabajando y que además reciben una buena jubilación (Kautonen, 2012). Además, existen adultos mayores que poseen una posición financiera bastante más sólida por haber acumulado riquezas durante su vida, situación que los deja en la buena posición de prescindir de fuentes de financiamiento externas, aunque este no es el caso más común en personas que viven en economías emergentes. (Kibler et al., 2012)

\section{Salud, costo de oportunidad del tiempo y desincentivos financieros}

A medida que las personas envejecen, como situación obvia, la salud comienza a deteriorase. Este fenómeno impacta los estilos de vida y obstaculiza los deseos de comenzar un negocio. Weber y Schaper (2004) concluyeron que los adultos mayores poseen bajos niveles de salud y energía para poder emprender. Además Singh y DeNoble (2003) logran establecer que cuando las personas envejecen y se les complica la salud, las actividades de ocio se vuelven más atractivas, afectado la motivación y predisposición a comenzar un negocio. Por otro lado, en muchos países se está introduciendo un seguro por enfermedad que permita romper con esta barrera. Dicha solución disminuye el temor de enfermarse y la inseguridad que surge frente a la posibilidad de ser incapaz de dar cumplimiento a los pagos que demande el negocio.

Interesante resulta mencionar que estudios realizados en algunos países desarrollados han demostrado que los adultos mayores se encuentran menos dispuestos y comprometidos a desarrollar actividades que den resultados a futuro o a plazo (Halabisky, 2012). Ellos prefieren realizar en esta etapa de sus vidas una mayor cantidad de actividades de ocio y valoran menos los beneficios que se pueden generar en el futuro en consideración a la incertidumbre de cosechar esos nuevos recursos (Levesque y Minniti, 2006). La situación se complica, aún más, cuando los beneficios estatales vinculados a ingresos por jubilación puede desincentivar a los adultos mayores a emprender. Lo anterior ocurre cuando un negocio puede no ofrecer suficientes ingresos en comparación con lo que se podría percibir por jubilación. (Halabisky, 2012).

Estudios realizados por Kibler et al. (2011) indican que las personas mayores temen perder sus beneficios sociales o de pensiones si obtienen ingresos en un negocio. Estudios de la Comisión Europea (2012) demuestran que el 59 \% de los adultos mayores de la Unión Europea, creen que no es beneficioso seguir trabajando más allá de los 55 años, debido a los beneficios del sistema de pensiones. De igual forma, estudios realizados por de Singh y DeNoble (2003) concluyeron que aquellos adultos mayores que disponen de altos niveles de recursos y riquezas, tienen menos interés de emprender, presentándose la riqueza como un desincentivo al emprendimiento.

\section{Contexto emprendedor y las instituciones relacionadas.}

De acuerdo a información de Halabisky (2012), una cantidad considerable de adultos mayores han pasado su vida completa trabajando en organizaciones e instituciones para recibir un sueldo, sin haber tenido conciencia de las oportunidades que se ofrecen para emprender.

Según Kibler et al. (2012), los adultos mayores tienen un enorme desafío en acceder a la información para desarrollar un negocio. Esto se debe a que dichos individuos no entienden la información publicada por los diferentes sitios gubernamentales o agencias de apoyo al emprendimiento, señalando que la información está destinada más bien a jóvenes emprendedores que a adultos mayores (Kibler et al., 2012). A su vez, de acuerdo a investigaciones realizadas por Kautonen (2012), los emprendedores de la tercera edad piden más claridad en las regulaciones a sus negocios, junto con mayor flexibilidad laboral y algunas reglas tributarias que sirvan como apoyo y no como barreras para poder crear y hacer crecer un negocio. De igual forma, y de acuerdo con Kibler et al. (2011), los adultos mayores no entienden los aspectos legales que les permite generar un negocio, de tal forma que deben solicitar más apoyo por parte de instituciones del Estado.

Cabe mencionar, de acuerdo con Kautonen (2008), que en ambientes sociales donde nunca ha existido experiencia emprendedora es probable que se presente una gran barrera para realizar un negocio. Según dicho autor, los emprendedores de la tercera edad, en múltiples oportunidades, sienten gran inseguridad sobre sus ambiciones empresariales cuando los amigos y la familia constantemente les aconsejan que la idea de iniciar un negocio es demasiado arriesgada. Los resultados de estas opiniones negativas, incluyendo la falta de apoyo financiero, social y la extensión del proceso para concretar el negocio, ocasionan que los adultos mayores renuncien a la idea de emprender. 
De acuerdo con Kautonen et al. (2009) un número considerable de emprendedores de la tercera edad trata de solucionar esta situación, refugiándose en nuevos amigos y en redes de contactos con el fin de obtener el apoyo emocional y la seguridad para llevar adelante una idea de un negocio.

\section{Educación emprendedora.}

Estudios realizados por Baucus y Human (1994) confirman que el apoyo a negocios de los adultos mayores puede ser de gran ayuda para la superación de ciertas barreras, principalmente para aquellos emprendedores de la tercera edad que lo hacen por primera vez y que no cuentan con un ingreso estable y están en situación de vulnerabilidad social y pobreza. Sin embargo, Kautonen (2012) concluye que, a pesar que numerosos emprendedores de la tercera edad sienten que poseen los conocimientos y habilidades para llevar adelante un negocio, mencionan que es muy difícil contar con buenos mentores y coachers. La falta de buenos mentores que conozcan la realidad propia de los adultos mayores constituye una lamentable barrera para emprender (Kautonen, 2012).

\section{Las asimetrías de condiciones entre adultos mayores y jóvenes en el emprendimiento.}

Para comenzar este apartado, es necesario explicar el significado de la comparación entre emprendedores de la tercera edad con su contraparte más joven. Hart et al. (2004), denomina "prime age" a emprendedores que poseen entre 20 y 49 años y "third age" a emprendedores de la tercera edad que poseen 50 años y más. Preliminarmente, se podría decir que cuando se comparan ambos grupos, el emprendimiento de la tercera edad podría visualizarse como una situación de poca importancia, sin embargo, este fenómeno no es marginal (Kautonen, 2009).

De acuerdo a diferentes estudios llevados a cabo por investigadores que compararon ambos grupos, se concluyó que existen diferencias sustanciales en varios aspectos, los cuales se describen a continuación. (Hart et al., 2004; Weber y Schaper, 2004; Kautonen, 2008; Kibler et al., 2011)

\section{Motivación, predisposición y desarrollo innovador.}

Estudios evidencian que la proporción de adultos mayores que están comenzando un negocio es la mitad en comparación a los jóvenes (Hart et al., 2004; Kautonen, 2008). Con dicho antecedente, se puede concluir que los emprendedores de la tercera edad, tienen menos motivación y predisposición para emprender (Curran y Blackburn, 2001; Hart et al., 2004). Sin embargo, la tasa de sobrevivencia en el largo plazo de un negocio fundado por emprendedores de la tercera edad, es mayor en comparación a su contraparte joven (Cressy y Storey, 1995). Estudios realizados por Kautonen y Luoto (2008) concluyeron que, después de 5 años, el 70\% de los negocios creados por adultos mayores se mantenían operando, mientras que aquellos fundados por jóvenes llegaban sólo al 28\%. Esta situación se explica, porque en la medida que las personas envejecen aumentan sus competencias y habilidades emprendedoras, pero la motivación tiende a decaer. (Rotefoss y Kolvereid, 2005).

Resulta importante afirmar que estudios realizados por Colovic y Lamonette (2012) sobre innovación establecieron que los adultos mayores son menos innovadores que su contraparte joven, esto referido tanto en innovación de productos como en innovación de procesos. Dicha situación se explica fundamentalmente porque los individuos de la tercera edad tienen problemas cognitivos que reducen la habilidad para crear productos innovadores, adoptar nuevas tecnologías y seguir capacitándose. De acuerdo con Botham y Graves (2009), los adultos mayores que innovan menos están en desventajas para crear nuevos negocios. Esta falta de innovación puede afectar el éxito de los negocios en el largo plazo. (Colovic y Lamonette, 2012)

\section{Capital humano, social y acceso a recursos financieros}

Estudios desarrollados por Weber y Schaper (2004) y Kautonen et al. (2008) concluyeron que los adultos mayores poseen más habilidades técnicas y administrativas que los emprendedores jóvenes. Por otra parte, las personas de más edad tienen los beneficios de la experiencia laboral que les permite enfrentar los problemas de mejor manera en comparación a personas no familiarizadas con habilidades de negocios (Kibler et al., 2011). Asimismo, el profundo conocimiento de una industria, que no poseen los jóvenes, puede ayudar a la persona a identificar las brechas para crear nuevos negocios (Kautonen, 2012).

Sin embargo, de acuerdo a estudios de Botham y Graves (2009), el riesgo para las personas mayores en la creación de una empresa puede ser objetivamente mayor en comparación a los jóvenes. Si el negocio llega a fracasar, estas personas tienen menos tiempo y oportunidades de empleo para contrarrestar las pérdidas del negocio, adquirir nuevas habilidades, reinventarse laboralmente y generar una fuente alternativa de ingresos (Roberts, 1991). Los jóvenes se apegan menos a los negocios, ya sea vendiéndolos o cambiando de giro, generándose una reinvención laboral y de capital humano en consideración a que tienen más tiempo para restablecerse en algún negocio posterior (Hart et al., 2004).

Por otro lado, en comparación con su contraparte más joven, los emprendedores de la tercera edad poseen redes de contactos más desarrolladas, las cuales han sido acumuladas en una buena cantidad durante su carrera laboral (Kibler et al., 2012). Estas mismas redes pueden, posteriormente, ser empleadas en sus propios negocios. Estudios desarrollados por Kibler et al. (2012) descubrieron que los emprendedores de la tercera edad que utilizan el capital social adquirido de un trabajo anterior tenían menos dificultades para iniciar y administrar su empresa, situación que no se presenta con los jóvenes emprendedores.

Un tema fundamental que aparece en la literatura, independiente del tipo de emprendimiento que se establezca, se refiere al adecuado apoyo o presencia de capital para la formación de un negocio. (Blanchflower y Oswald, 1998; Lussier y Pfeifer, 2001). De acuerdo con Kibler et al. (2011), los adultos mayores enfrentan las mismas dificultades para conseguir recursos financieros en comparación con emprendedores más jóvenes. Sin embargo, en países desarrollados existen adultos mayores que poseen una posición financiera bastante más sólida que los emprendedores jóvenes, en consideración a las riquezas acumuladas durante su vida, que facilitan la formación de un negocio (Parker, 2001). 


\section{Contexto emprendedor e impacto económico}

De acuerdo a estudios realizados por Pilkova et al. (2014), se evidencia que la burocracia pública y carga fiscal vía impuestos podría tener un efecto más fuerte en desalentar la actividad emprendedora en la tercera edad, en comparación con la población más joven. Esta situación puede ser causada por una menor capacidad de las personas mayores de adaptarse a los entornos cambiantes y superar las barreras burocráticas estatales (Weber y Schaper, 2004).

Interesante es tener presente que investigaciones realizadas por Zhang (2008) establecen la existencia de altas tasas de autoempleo en adultos mayores, que contribuyen en forma importante al crecimiento económico. A su vez, los estudios de Curran y Blackburn (2001) concluyeron que los emprendedores de la tercera edad contribuyen en menor medida a la creación y contratación de empleo y, si esto llega a suceder, lo concretan en menor número de personas en relación a emprendedores más jóvenes. Lo expresado se vincula directamente con estudios realizados por Kautonen et al. (2013) que confirman el aserto que las personas mayores son más propensas a auto emplearse en comparación a su contraparte más joven.

\section{Salud y niveles de educación}

En relación a la salud y bienestar, estudios concluyeron que los adultos mayores tienen mayores desafíos que su contraparte joven para emprender, principalmente por los bajos niveles de energía y problemas de salud (Weber y Schaper, 2004). Estos factores perjudican notablemente el emprendimiento debido a que generan dificultades para las personas de la tercera edad cuando se trata de entregar el esfuerzo y la energía destinadas a hacer surgir sus negocios (Staudinger, 1999).

Weber y Schaper (2004) señalan que los adultos mayores poseen niveles de educación más bajos en relación a emprendedores jóvenes. Según Parker (2004), los emprendedores de la tercera edad no necesariamente buscaron un grado universitario para emprender. Este argumento en general puede estar afectando niveles más bajos de educación, principalmente para aquellos emprendedores de la tercera edad que comenzaron sus primeros negocios en forma muy temprana y que ya se encuentran establecidos. (Kautonen, 2008)

\section{Programas de fomento y apoyo al emprendimiento del adulto mayor.} De acuerdo con esta investigación, cada día en el mundo existen nuevos programas para potenciar el emprendimiento del adulto mayor y ayudar a generar nuevas fuentes de ingresos (Halabisky, 2012).

A continuación se describen algunos de estos programas:

\section{Programas para promover los beneficios del emprendimiento.}

Un programa desarrollado en Europa que focaliza exclusivamente en mujeres de la tercera edad se denomina "Female Proyect". Este programa ofrece capacitación y educación emprendedora en países tales como el Reino Unido, Holanda, Chipre y Malta y tiene apoyo de la Unión Europea y de empresas privadas. Dicha iniciativa apoya a las mujeres de la tercera edad, con el objeto de darles facilidades para comenzar su propio negocio. Básicamente, es una plataforma online que incluye un set de herramientas, ofreciendo coaching y mentoring para promover un emprendimiento.

Un segundo programa, desarrollado por la Comisión Europea (2012), fue el proyecto "Grundtvig". Este proyecto tenía como objetivo promover historias personales de emprendedores de la tercera edad con fin de dar a conocer el emprendimiento como una opción laboral (Halabisky, 2012).

Un tercer proyecto, también desarrollado en Europa, fue el denominado "Memoro", cuyo objetivo fue recoger diferentes historias de empresarios de edad avanzada y hacer cortometrajes para promover el espíritu empresarial entre personas mayores (Best Agers, 2011).

\section{Programas para el desarrollo de nuevas habilidades.}

Un primer programa que fue financiado a través del Fondo Social Europeo (FSE) en Hungría, promovía la integración de las personas de la tercera edad desfavorecidas en el mercado laboral, utilizando estrategias de emprendimiento y de esquemas de capacitación para ayudarlos a comenzar un negocio.

Un segundo proyecto, que se desarrolla en Canadá, llamado "Tiow”, tiene como objetivo capacitar a trabajadores de edad avanzada y en condiciones de vulnerabilidad social y pobreza para que puedan desarrollar sus propios emprendimientos. Esta iniciativa ofrece servicios de asistencia para mejorar habilidades emprendedoras y potenciar la experiencia empresarial (Halabisky, 2012).

Un tercer programa que se desarrolla en Australia, denominado "Neis", es orientado a adultos mayores sobre 50 años que tienen problemas sociales y sufren discriminación por la edad para comenzar un negocio. Dicha iniciativa está financiada por el gobierno de Australia desde el año 2006 y entrega por siete semanas capacitación y educación emprendedora. Sin perjuicio de anterior, después de ese período brinda por doce meses coaching y mentoría uno a uno (Kautonen, 2012).

Programas para el desarrollo de redes y acceso a financiamiento. Un primer proyecto llamado "Biiugi", desarrollado en Alemania, ofrece una plataforma en línea que permite a emprendedores de la tercera se edad conectarse con empresarios más experimentados con el objeto de obtener el asesoramiento y compartir experiencias.

Otra iniciativa desarrollada en Estados Unidos de Norteamérica es el programa "Score" que fue lanzado hace varias décadas para ayudar a los militares retirados a orientarlos y capacitarlos en administración de negocios. Uno de los servicios que aporta "Score" es ayudar a los empresarios de la tercera edad a encontrar mentores a través de una base de datos en línea y facilitar tutorías uno a uno. Este programa posee cerca de 9 millones de clientes (Kautonen, 2012).

En el Reino Unido, el programa "Prime" de la Fundación del Príncipe de Gales, entrega educación y acceso a financiero a adultos mayores de 50 años, que se encuentren desempleados o en situación de pobreza. Creado en 1998, apoya a adultos mayores desempleados y jubilados para que comiencen sus propios negocios. Proveen de información, 
talleres, educación y capacitación, redes de contacto, coaching y préstamos (micro finanzas) a un costo muy bajo (Kautonen y Luoto, 2008)

Otro proyecto llamado "Mature Entrepreneur Project", desarrollado en Polonia, tiene como objetivo apoyar la iniciativa empresarial para los mayores de 50 años con el objeto de mantener su negocio funcionado o bien comiencen uno. Los participantes reciben aportes en dinero para ayudarles a iniciar un negocio y también reciben capacitación y asesoramiento empresarial. Este proyecto fue llevado a la televisión para fomentar y promover el emprendimiento de los adultos mayores en ese país (Halabisky, 2012)

\section{Discusión}

Tomando en cuenta la revisión de la literatura y los cambios demográficos que se están generando, se puede apreciar que las personas de la tercera edad serán cada vez más importante en nuestra sociedad. Uno de los grandes desafíos de este nuevo escenario se refiere a tratar de aminorar de modo eficiente las situaciones de discriminación por parte de la sociedad que sufren los adultos mayores que aún se encuentran en buenas condiciones físicas y mentales.

En efecto, la marginación de la vida laboral activa constituye una realidad que sufren estas personas, impidiéndoles tener posibilidades de integrarse vitalmente al mercado laboral. Los referidos problemas de discriminación son significativos.

Surge, entonces, el emprendimiento, no sólo como una alternativa viable para mejorar los ingresos de las personas mayores y mejorar su calidad de vida, sino también como remedio para aminorar la desintegración del adulto mayor de la vida laboral en una sociedad. Para facilitar el entorno emprendedor de los adultos mayores se plantean las siguientes propuestas:

En primer lugar, es fundamental que el gobierno, con la ayuda de las agencias del estado, pueda intervenir a fin de mantener a las personas mayores en la fuerza laboral. En efecto, si personas de este grupo etario pierden sus trabajos, existen altos riesgos de vulnerabilidad social. Como sabemos y ya se ha expresado en este trabajo, los adultos mayores poseen un valioso capital humano acumulado a través de parte importante de sus vidas, que puede llegar a utilizarse para realizar actividades emprendedoras en años posteriores. Por otro lado, respecto de aquellas personas que ya están jubiladas, resulta imperioso incentivarlas y capacitarlas a fin de obtener toda la riqueza basada en su experiencia laboral, profesional y social que estos adultos pueden ofrecer al generar emprendimientos de diversa índole.

Se propone crear en Chile el Año del envejecimiento activo, de tal manera de crear conciencia en la sociedad que las personas de la tercera edad, hoy por hoy, buscan un envejecimiento dinámico, siendo el emprendimiento una herramienta que les permite tener un balance de trabajo, ingresos y tiempo libre. Esta política ayudaría a mejorar la apertura cultural en Chile hacia los adultos mayores para incentivarlos en actividades emprendedoras y no se sientan discriminados.

En segundo lugar, respecto de aquellos adultos mayores que actualmente no tienen jubilación o bien que su principal fuente de ingresos es la contribución de sus hijos o de sus familias, el emprendimiento es una opción atractiva para obtener una renta. El desarrollo de actividades empresariales y la conversión desde el trabajo dependiente al emprendimiento parece mejorar las alternativas de superación de las personas. (Kornfeld, 2013). A su vez, el emprendimiento también constituye una herramienta útil respecto de aquellos los adultos mayores que tienen una mejor posición económica y que lograron ahorrar más durante su vida laboral activa, puesto que no tienen totalmente asegurados sus ingresos, debido a los fuertes cambios que experimentan los mercados financieros.

Actualmente, el gobierno chileno ha estado siempre apoyando el entorno y la iniciativa emprendedora. Una gran cantidad de oportunidades para futuros empresarios están empezando a surgir en Chile. El gobierno no puede confiar solo en apoyar iniciativas emprendedoras en jóvenes. Creemos que ya es momento de mirar a los adultos mayores como futuros emprendedores y brindarles la oportunidad de mejorar los ingresos y fomentar, en lo posible, el desarrollo de negocios innovadores.

Para lo anterior, es necesario generar programas de apoyo, a fin de fomentar esta iniciativa y superar obstáculos, de la misma manera como se realiza en otras partes del mundo.

Resulta de alta relevancia no visualizar el apoyo a estos emprendedores como simples programas asistenciales.

Hoy en día, no existen en Chile programas de emprendimiento destinados exclusivamente a personas naturales de la tercera edad que apoyen y brinden seguimiento para comenzar un negocio. También presenciamos la carencia de buenos "coachers" y mentores que conozcan la realidad propia de un emprendedor de la tercera edad.

El Estado de Chile podría crear en algunas instituciones que fomentan el emprendimiento, tales como CORFO o SERCOTEC, junto con el SENAMA, programas destinados exclusivamente a apoyar a emprendedores en este grupo etario. Evidencias empíricas muestran que apoyar el emprendimiento del adulto mayor no solo ayuda a este grupo, sino también es la sociedad entera la que obtiene un notable provecho.

En tercer lugar, se propone un modelo de intervención para fomentar la motivación, salud y el desarrollo de nuevas habilidades. Esta propuesta busca incentivar el lanzamiento de nuevas empresas por parte de adultos mayores. La vejez trae, sin duda, un declive biológico y físico. La inactividad plantea un riesgo para que se generen diversas enfermedades crónicas. Por dicha razón, las personas de la tercera edad deberían mantenerse ocupadas, siendo el emprendimiento una opción para equilibrar sus vidas.

La idea de este proyecto es incentivar a los adultos mayores a formar parte de incubadoras de negocios hechas a la medida para la creación 
de nuevos emprendimientos. De esta forma, los conocimientos y la carrera laboral de este grupo etario podrían concretarse en buenas ideas para ser llevadas a la práctica.

Otro objetivo importante de esta propuesta se refiere a la creación de nuevas fuentes laborales e ingresos, la posibilidad de crear negocios familiares que permitan trascender a nuevas generaciones, de desarrollar nuevas habilidades y mejorar la calidad de vida. Por otro lado, permitirá en Chile generar nuevos conocimientos sobre el tema del emprendimiento en el adulto mayor.

En cuarto lugar, existen evidencias empíricas que sitúan a los adultos mayores en mejor posición para emprender que otros segmentos de la población, a pesar de todas las barreras que se presentan. Estas personas poseen experiencia laboral, capital social y capital humano, entre otros factores, que puede ser utilizado para emprender. También se debe considerar que los negocios fundados por adultos mayores tienen una tasa de sobrevivencia más alta que otros segmentos de la población, permitiendo desarrollar alternativas de negocios más viables en el largo plazo.

Se propone también la elaboración de programas destinados a que un cierto segmento de adultos mayores con antecedentes profesionales de alto nivel y que no requieren o no buscan emprender un negocio, puedan cumplir el rol de "coach" de negocios o mentor, con el fin ser colaboradores en el desarrollo de emprendimientos respecto de otros adultos mayores que requieren de su ayuda.

Asimismo, algunos adultos mayores pueden ayudar a sus coetáneos en otra dirección. En efecto, se trata de individuos más ricos, y en esta situación, se propone establecer un plan para que actúen como inversionistas ángeles y de este modo se involucren en los equipos de trabajo de emprendedores de tercera edad.

Finalmente, apoyar el emprendimiento del adulto mayor tiene un fuerte impacto en el desarrollo económico un país (Zhang, 2008).

En esta línea de acción, se manifiesta la urgente necesidad de planificar a nivel gubernamental y privado todas las directrices que permitan ubicar a los adultos mayores como nuevos emprendedores y, desde ese lugar, contribuir al desarrollo económico de Chile.

\section{Conclusiones}

Considerando los cambios demográficos de nuestra sociedad, se puede concluir que los adultos mayores tendrán cada vez más un rol más importante. Uno de los grandes desafíos que se presentan es mantener a estas personas activas y con buena calidad de vida. Por ello se propone al emprendimiento como una alternativa para contrarrestar la falta de oportunidades laborales y de ingreso.

Como sociedad no es apropiado olvidar que los adultos mayores pueden aún contribuir económicamente al país. Muchos de ellos se encuentran en buenas condiciones físicas y mentales, además tienen la motivación, la necesidad y la energía para participar y beneficiarse de un emprendimiento.
El gobierno de Chile debe iniciar el viaje de valorar y fomentar el emprendimiento del adulto mayor como una estrategia nacional, no solo como una medida destinada al bienestar personal de este grupo etario, sino también como estrategia para el desarrollo económico del país.

Generar programas de apoyo y fomento para esta iniciativa, resulta una medida adecuada, principalmente para aquellos adultos mayores que están en desventaja económica y que no pueden encontrar un trabajo.

Finalmente cabe hacer presente que el estudio del emprendimiento del adulto mayor es aún una disciplina en desarrollo e indudablemente tendrá en el futuro una creciente importancia para la investigación.

\section{Referencias}

Arkebauer, J. B. (1995). Golden Entrepreneuring: The Mature Person's Guide to a Successful Business. NY: Mcgraw-Hill.

Best Agers (2011), Newsletter No. 4, pp. 1-10.

Blackburn, R., Hart, M. y O'reilly, M. (2000). Entrepreneurship in the Third Age: New Dawn or Misplaced Expectations? 23rd ISBA National Small Firms Policy and Research Conference, Aberdeen University.

Blackburn, R., Mackintosh, L. y North, J. (1998), Entrepreneurship in the Third Age, Kingston University Small Business Research Centre, Surrey.

Blanchflower, D. y Oswald, A. J. (1998). What Makes an Entrepreneur? Journal of Labour Economics, 16 (1), 26-59. http:// dx.doi.org/10.1086/209881

Baucus, D. A. y Human, S.D. (1994). Second-career entrepreneurs: A multiple case study analysis of entrepreneurial processes and antecedent variables. Entrepreneurship Theory and Practice, 19 (2), 41-71.

BIS Department of Business and Innovation Skills (2011). Phasing out the Default Retirement Age: Government Response to Consultation. London: BIS.

Botham, R. y Graves, A. (2009). Third age entrepreneurs: Innovative business start ups in mid-life and beyond- understanding the drivers and removing the barriers. Interim Report to NESTA.

Canals, B. (2012). Contexto socio demográfico del envejecimiento en Chile. In. Academia Parlamentaria Cámara de Diputados de Chile (Eds.), Cuadernillos Hemiciclos (pp.50-55). Valparaíso: Cámara de diputados.

Clark, G., Munnell, A. y Orszag, J. (2006). The Oxford Handbook of Pensions and Retirement Income. OUP:Oxford. http://dx.doi. org/10.1093/oxfordhb/9780199272464.001.0001

Colovic, A. y Lamonette, O. (2012) Are third age entrepreneurs innovative? Evidence from GEM data. Revue Française de Gestion, 227, 127-141. http://dx.doi.org/10.2139/ssrn.2327021 
Comisión Europea, (2012), Active Ageing, Special Flash Eurobarometer No. 378, pp.1-12.

Cressy, R. y Storey, D.J. (1995). New Firms and Their Banks. Warwick University Business School and NatWest.

Curran, J. y Blackburn, R. (2001). Older people and the enterprise society: age and self-employment propensities. Work, employment and society, 15 (4), 889-902. http://dx.doi. org/10.1177/095001701400438279

De Bruin, A., y Firkin, P. (2001). Self-employment of the older worker. Labour Market Dynamics Research Programme, Massey University.

Fernandez, L. S., y Rey, E. F. (2010). Competencias emprendedoras y desarrollo del espíritu empresarial en los centros educativos. Educación 21, 13(1). http://dx.doi.org/10.5944/educxx1.13.1.275

Froud, J., Haslam, C., Johal, S.,y Williams, K. (2002) Financialisation and the coupon pool. Capital and Class, 26, 119-151. http://dx.doi. org/10.1177/030981680207800106

Fuchs, V.C. (1982). Self-employment and labour force participation of older males, Journal of Human Resources, 17(3), 339-357. http:// dx.doi.org/10.2307/145584

Halabisky, D. (2012). Entrepreneurial Activities in Europe - Senior Entrepreneurship. OECD Employment Policy Papers. http://dx.doi. org/10.1787/5jxrcml7lhxq-en

Hart, M., Anyadike-Danes, M. y Blackburn, R. (2004). Entrepreneurship and age in the UK: Comparing Third Age and Prime Age new venture creation across the regions. Paper presented at RENT XVIII, Copenhagen.

Hinz, T., y M. Jungbauer-Gans (1999). Starting a Business after Unemployment: Characteristics and Chances of Success - Empirical Evidence from a Regional German Labour Market. Entrepreneurship and Regional Development, 11(4), 317-333. http://dx.doi. org/10.1080/089856299283137

Kadefors, R. (2011), Costs and Benefits of Best Agers Employment, Best Agers Project Retrieved from http://www.best-agers-project.eu/

Karoly, L. A., y Zissimopoulos, J. (2004). Self-employment and the $50+$ population.

Kautonen, T.; Down, S. y South, L. (2008). Enterprise support for older entrepreneurs: the case of Prime in the UK. Entrepreneurship and Regional Development, 14 (2), 85-101. http://dx.doi. org/10.1108/13552550810863071

Kautonen, T. (2008). Understanding the older entrepreneur: Comparing third age and prime age entrepreneurs in Finland. International Journal of Business Science and Applied Management, 3(3), 3-13.
Kautonen, T., y Luoto, S. (2008). Entrepreneurial intentions in the third age: The impact of career history. In University of Vaasa.

Kautonen, T., Tornikoski, E. y Kibler, E. (2009). Entrepreneurial intentions in the third age: the impact of perceived age norms. Small Business Econonomy, 37, 219-234. http://dx.doi. org/10.1177/0266242610368592

Kautonen, T.; Down, S.; Welter, F.; Vainio, P.; Palmroos, J.; Althoff, K. y Kolb, S. (2010). Involuntary self-employment as a public policy issue: a cross-country European review. International Journal of Entrepreneurial Behaviour \& Research, 16 (2), 112-129. http://dx.doi. org/10.1108/13552551011027002

Kautonen, T., Van Gelderen, M. y Torniskoski, E. T. (2011). Predicting entrepreneurial behaviour: a test of the theory of planned behaviour. Applied Economics, 45 (6), 697-707. http://dx.doi.org/10.1080/00036 846.2011.610750

Kautonen, T. (2012). Senior Entrepreneurship. A background paper for the OECD centre for entrepreneurship. Smes and local development. Paris, France: OECD.

Kautonen, T., Down, S. y Minniti, M. (2013). Ageing and entrepreneurial preferences. Small Business Economics, 42(3), 579594. http://dx.doi.org/10.1007/s11187-013-9489-5

Kibler, E., Wainwright, T., Kautonen, T., \& Blackburn, R. (2011). (Work) Life after work: understanding barriers to older entrepreneurship in London. In ICSB World Conference Proceedings. International Council for Small business (ICSB).

Kibler, E., Wainwright, T., Kautonen, T.. y Blackburn R. (2012). Can social exclusion against older entrepreneurs be managed? Journal of Small Business Management. http://dx.doi.org/10.1111/jsbm.12194

Kornfeld, R. (2013). Chile y sus mayores, bienestar general en la vejez. In. Senama (Ed.) Encuesta calidad de vida y vejez, (pp. 19-20). Santiago de Chile: Pontificia Universidad Católica de Chile.

Levesque, M., y Minniti, M. (2006). The Effect of Aging on Entrepreneurial Behavior, Journal of Business Venturing, 21(2), 177194. http://dx.doi.org/10.1016/j.jbusvent.2005.04.003

Levie, J. (2014). Global Entrepreneurship Monitor: Scotland 2013 Retrieved form http://www.gemconsortium.org/docs/download/3348

Loretto, W. (2010). Work and retirement in an ageing world: the case of older workers in the UK. Twenty-First Century Society, 5(3), 279294. http://dx.doi.org/10.1080/17450144.2010.480831

Loretto, W. y White, P. (2006). Employers attitudes, practices and policies towards older workers. Human Resource Management Journal, 16, 313-330. http://dx.doi.org/10.1111/j.1748-8583.2006.00013.x

Lussier, R. y Pfeifer, S. 2001, A Crossnational Prediction Model of Business Success, Journal of Small Business Management, 39 (3), 228-239. http://dx.doi.org/10.1111/0447-2778.00021 
Maestas, N. y Zissmopoulos, J. (2010). How Longer Working Lives Ease the Crunch of Population Ageing. The Journal of Economic Perspectives, 24 (1), 139-160. http://dx.doi.org/10.1257/jep.24.1.139

McClelland, E., Swail, J., Bell, J., \& Ibbotson, P. (2005). Following the pathway of female entrepreneurs. International Journal of Entrepreneurial Behavior \& Research, 11(2), 84-107. http://dx.doi. org/10.1108/13552550510590527

Mckay, R. (2001). Women entrepreneurs: Moving beyond family and flexibility.InternationalJournalofEntrepreneurialBehaviour\& Research, 7 (4), 148-165. http://dx.doi.org/10.1108/13552550510590527

Mrva, M., y Stachová, P. (2014). Regional development and support of SMEs-how university project can help. Procedia-Social and Behavioral Sciences, 110, 617-626. http://dx.doi.org/10.1016/j.sbspro.2013.12.906

Munnel, A., Sunden, A., Taylor, C. (2002) What determines 401(k) participation and contributions? Social Security Bulletin, 64, 64-75. http://dx.doi.org/10.2139/ssrn.253037

North, D.C (1990). Institutions, Institutional Change and Economic Performance. Cambridge: Cambridge University Press.

Oecd (2001). Fiscal Implications of Ageing: Projections of AgeRelated. OECD Economic Outlook, 69 (4), 145-167.

Oecd (2006). Live Longer, Work Longer. Paris: OECD.

Oecd (2012). Policy Brief on Senior Entrepreneurship. Entrepreneurial Activities in Europe Retrieved from http://ec.europa.eu/social/ BlobServlet?docId=9644\&langId=en

Oecd (2012). Policy Brief on Senior Entrepreneurship. Luxembourg: European Commission.

Oms (Organización Mundial de la Salud) (2012). La buena salud añade vida a los años Retrieved from http://apps.who.int/iris/ bitstream/10665/75254/1/WHO_DCO_WHD_2012.2_spa.pdf.

Parker, S.C. (2001). Prosperity not poverty: An investigation into the holdings and distribution of wealth of older British entrepreneurs. University of Durham, Department of Economics and Finance.

Parker, S..C. (2004). The Economics of Self-Employment and Entrepreneurship. Cambridge: Cambridge University Press.

Pilkova, A., Holienka, M. y Rehak, J. (2014). Senior entrepreneurship in the perspective of European entrepreneurial environment. Procedia Economics and Finance, 12, 523 - 532. http://dx.doi.org/10.1016/ s2212-5671(14)00375-X
Roberts, E.B. (1991). Entrepreneurs in High Technology: Lessons from MIT and Beyond. Oxford: University Press.

Rotefoss, B. y Kolvereid, L. (2005). Aspiring, nascent and fledgling entrepreneurs: An investigation of the business start-up process. Entrepreneurship and Regional Development, 17 (2), 109-127. http:// dx.doi.org/10.1080/08985620500074049

Senama, Servicio nacional del adulto mayor (2011). Encuesta nacional de inclusión y exclusión social del adulto mayor en Chile, Gobierno de Chile Retrieved form http://www.senama.cl/filesapp/SEGUNDA\%20 ENCUESTA.pdf.

Senama, Servicio nacional del adulto mayor (2013). Resultados Tercera Encuesta Nacional Calidad de Vida en la Vejez Retrieved from http://www.senama.cl/filesapp/Chile\%20y\%20sus\%20mayores\%20 2013,\%20Encuesta\%20de\%20Calidad\%20de\%20Vida.pdf].

Singh y De Noble, A. (2003). Early Retirees as the Next Generation of Entrepreneurs. Entrepreneurship Theory and Practice, Vol. 23 (3), 207-226. http://dx.doi.org/10.1111/1540-8520.t01-1-00001

Staudinger, U. M. (1999). Older and Wiser? Integrating results on the relationship between age and wisdom-related performance. International Journal of Behavioral Development, 23 (3), 641-664. http://dx.doi.org/10.1080/016502599383739

Wainwright, T y Kibler, E. (2013). Beyond financialization: older entrepreneurship and retirement planning. Journal of Economic Geography, 14 (4), 849-864. http://dx.doi.org/10.1093/jeg/lbt023

Weber, P., y Schaper, M. (2004). Understanding the Grey Entrepreneur. Journal Enterprising Culture, 12 (2), 147-164. http:// dx.doi.org/10.1142/s0218495804000087

Webster, B. y Walker, B. (2005). Smart training for the older entrepreneur. Presented at the International Council of Small Business World Conference, Melbourne, June 2005.

Zhang, T. (2008). Elderly Entrepreneurship in an Aging US Economy. Series on Economic Development and Growth. http://dx.doi. org/10.1142/6852

Zissmopoulos, J. M., y Karoly, L. A. (2007). Transitions to selfemployment at older ages: The role of wealth, health, health insurance and other factors. Labour Economics, 14 (2), 269-295. http://dx.doi. org/10.1016/j.labeco.2005.08.002 